Localizador: 19019 doi: $10.35366 / 91754$

\title{
Uso actual de la vitamina D en dermatología
}

\author{
Current use of vitamin D in dermatology \\ Ramón Adrián García-Galaviz, * José Manuel Díaz-González, \\ Luis Enrique Cano-Aguilar, ${ }^{\S}$ Judith Domínguez-Cherit
}

Palabras clave:

Vitamina D, dermatología, metabolismo mineral.

Keywords: Vitamin D, dermatology, mineral metabolism.

* Residente de Dermatología.

* Dermatooncólogo y

Cirujano Dermatólogo.

${ }^{\S}$ Médico Cirujano.

"Jefa del Departamento de

Dermatología.

Instituto Nacional de Ciencias Médicas y Nutrición «Salvador Zubirán».

Conflicto de intereses: Ninguno.

Recibido:

26/Abril/2019.

Aceptado:

02/Septiembre/2019

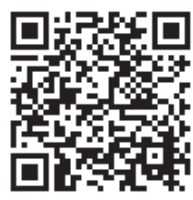

\begin{abstract}
REsUMEN
La vitamina $\mathrm{D}$ es una provitamina liposoluble de la hormona esteroidea 1,25- hidroxivitamina D3 sintetizada en los queratinocitos, producida por vía endógena ante la exposición a los rayos ultravioleta o por vía exógena mediante suplementación. La producción de la vitamina D está determinada por el fototipo de Fitzpatrick, el sexo, el índice de masa corporal y los polimorfismos en el receptor de vitamina D. En épocas anteriores, la vitamina D era considerada una hormona con función exclusivamente relacionada con el metabolismo y la homeostasis del calcio. En la actualidad, ha sido vinculada con diversas patologías de carácter neoplásico, autoinmune, cardiovascular y dermatológico. En el área de la Dermatología, la alteración de la vitamina $D$ desempeña un papel esencial en la etiopatogenia de diferentes dermatosis de carácter inflamatorio y neoplásicas, se encuentra relacionada con la gravedad de presentación, pronóstico a corto y largo plazo, así como recurrencia de éstas. Por lo tanto, conocer las principales dermatosis relacionadas con la alteración en la regulación inmunitaria de la vitamina D es de suma importancia para el manejo y posible suplementación como tratamiento coadyuvante de la enfermedad. Consideramos que la suplementación de vitamina D es útil para mantener la homeostasis celular de la piel.
\end{abstract}

\section{ABSTRACT}

Vitamin $D$ is a liposoluble provitamin of the steroid hormone 1,25-hydroxyvitamin D3 synthesized in keratinocytes, produced by the endogenous route when exposed to UV rays or exogenously by means of supplementation. The production of vitamin $D$ is determined by Fitzpatrick's phototype, gender, body mass index and polymorphisms in the vitamin $D$ receptor. Previously, it was considered a hormone with function exclusively related to the calcium metabolism and homeostasis. Currently, the role of the hormone has been linked to an increasing number of neoplastic, autoimmune, cardiovascular and dermatological diseases. In Dermatology, altered vitamin D plays an essential role in pathogenesis of different inflammatory and neoplastic dermatoses, it is related to severity, short and long-term prognosis, as well as recurrence of the disease. Therefore, knowing the main dermatological diseases related to altered immune regulation of vitamin $D$ is of utmost importance for the management and possible supplementation as an adjuvant treatment. We consider that Vitamin D supplementation is useful maintaining cell homeostasis of the skin.

\section{INTRODUCCIÓN}

L a vitamina $\mathrm{D}$ es una provitamina liposoluble de la hormona esteroidea 1.25-hidroxivitamina D3 sintetizada fotoquímicamente en los queratinocitos. Su precursor primario, el 7 hidroxicolesterol, es modificado por la luz ultravioleta B (UVB) dentro del espectro de 280 a 315 nm por vía artificial, por vía solar o por consumo exógeno, produciendo calcitriol, dependiendo de la cantidad de exposición. ${ }^{1}$ Éste se hidroxila en el hígado y el riñón por las enzimas 25 -hidroxilasa y $1 \alpha$-hidroxilasa, respectivamente, produciendo la forma activa de la hormona. La producción de la vitamina $\mathrm{D}$ está determinada por el fototipo de Fitzpatrick, el sexo, el índice de masa corporal (IMC) y los polimorfismos en el receptor de vitamina D. El nivel plasmático de 25-hidroxivitamina D se considera el mejor indicador del estatus de vitamina $D$, ya que refleja las contribuciones de la síntesis cutánea y la ingesta dietética. ${ }^{2}$ Esta vitamina ha sido relacionada con diversas patologías neoplásicas, dermatológicas, autoinmunes, trastornos esqueléticos y cardiovasculares. ${ }^{3}$ En Dermatología, el nivel de vitamina D se encuentra alterado en cáncer de piel melanoma y no melanoma, psoriasis, ictiosis, vitíligo, enfermedades ampollosas, esclerodermia, lupus eritematoso sistémico, dermatitis atópica, acné, alopecia y fotodermatosis. ${ }^{3}$

La vitamina $\mathrm{D}$ desempeña un papel primordial en la homeostasis y en el metabolismo del calcio. En la actualidad ha sido reconocida 
como una hormona esencial en diversos procesos metabólicos independientes del calcio, siendo relevante su función inmunorreguladora, de particular importancia en las enfermedades dermatológicas. Su rol como modificador de la fisiopatología de la piel es complejo. Sus receptores se expresan en queratinocitos de la capa basal y espinosa, donde promueve la síntesis de queratina, involucrina, transglutaminasa, filagrina y loricrina. Estas proteínas permiten la cornificación del queratinocito y simultáneamente inhiben la proliferación alterada de los mismos. ${ }^{4}$ Las funciones de la vitamina $\mathrm{D}$ en la epidermis incluyen la proliferación, la diferenciación y la apoptosis de los queratinocitos, así como la respuesta inmunitaria cutánea.

Estudios epidemiológicos y experimentales mencionan un rol primordial de la vitamina $\mathrm{D}$ en la mortalidad asociada al cáncer debido a que suprime la proliferación celular, disminuye las alteraciones de las células tumorales e inhibe la angiogénesis en el tejido neoplásico.

La vitamina D y su receptor (VDR) regulan el procesamiento de las cadenas largas de glucosilceramidas que forman la barrera cutánea e inician la respuesta inmunitaria innata cutánea, al inducir el receptor tipo toll (TLR) 2, lo que producirá catelicidina, resultando en actividad antimicrobiana. ${ }^{4}$

Los monocitos promueven la activación localizada de la vitamina $\mathrm{D}$ en respuesta a patógenos, así como la inducción de la producción de catelicidina. Las variaciones del nivel sérico de vitamina $\mathrm{D}$ correlacionan con la inducción de la producción de catelicidina por los monocitos. Por lo tanto, individuos con niveles séricos bajos de vitamina $\mathrm{D}$ tendrán menor capacidad de mostrar una respuesta antimicrobiana adecuada y están en mayor riesgo de sufrir infecciones. Por el contrario, al suplementarla en individuos deficientes ha demostrado mejorar la inducción de producción catelicidina de monocitos mediada por TLR y podría tener un efecto protector contra infecciones. ${ }^{7}$

Las células dendríticas y macrófagos expresan VDR y la suplementación de vitamina $\mathrm{D}$ ha demostrado que inhibe la maduración de las primeras, suprime la presentación de antígenos y promueve una respuesta celular T tolerogénica. ${ }^{4}$

Los linfocitos $\mathrm{T}$ cooperadores $(\mathrm{TH})$ parecen ser el principal objetivo de la vitamina $\mathrm{D}$ porque suprime su proliferación y modula la producción de citocinas. Estudios experimentales han demostrado que la vitamina $D$ inhibe la vía $\mathrm{TH} 1$, promueve TH 2, y suprime la producción de IL-17. Las células T reguladoras (Treg), que actúan como inmunosupresores para prevenir autoinmunidad o respuestas inmunitarias exhuberantes, son fuertemente inducidas por la vitamina D. ${ }^{4,8}$

El objetivo del presente artículo es revisar la evidencia que respalda el uso de la vitamina $\mathrm{D}$ en las diversas dermatosis y que resulta esencial en el manejo de los pacientes para una mejoría clínica y pronóstica.

\section{TIPOS DE DERMATOSIS}

\section{Quemadura solar}

La quemadura solar se caracteriza por un flujo aumentado de células inflamatorias a la piel con la liberación de factor de necrosis tumoral (TNF) a y producción excesiva de sintasa de óxido nítrico inducible (iNOS) como resultado de la exposición prolongada a la radiación UV. La vitamina $\mathrm{D}$ se ha relacionado con una disminución del daño celular al suprimir citocinas proinflamatorias como el TNF $\alpha$ y la iNOS a través de la arginasa 1 , un mediador antiinflamatorio producido por macrófagos activados por el VDR. Scott y colaboradores realizaron un estudio aleatorizado, doble ciego donde administraron 200,000 UI de vitamina D vía oral a 20 pacientes adultos sanos una hora después de una quemadura solar producida por radiación ultravioleta, con posterior toma de biopsia a las 48 horas. La dosis mencionada de vitamina $\mathrm{D}$ probó reducir el eritema y la inflamación resultante al disminuir TNF $\alpha$ e iNOS en el corte histológico. ${ }^{9}$ Sin embargo, no ha sido demostrado que un nivel sérico de vitamina $\mathrm{D}$ adecuado prevenga o disminuya la quemadura solar. Es posible que la vitamina D limite la carcinogénesis inducida por la radiación UV al promover el mecanismo de defensa celular y restaurar su función de barrera al eliminar los queratinocitos con daño genético. ${ }^{10}$

\section{Psoriasis}

El nivel sérico de la vitamina $\mathrm{D}$ ha sido relacionado con la etiopatogenia, duración y gravedad de la psoriasis medida por el índice de gravedad y área de psoriasis (PASI). ${ }^{11,12}$ La deficiencia de vitamina $D$ en la psoriasis podría explicarse por: la disminución en la exposición solar, el uso de medicamentos que interfieren con su metabolismo o una ingesta deficiente de la misma. Por otro lado, la psoriasis es considerada una enfermedad inflamatoria autoinmune que al ser tratada con vitamina $\mathrm{D}$ se modula la expresión de las citoqueratinas 1 y 10 en el estrato espinoso, las cuales se encuentran alteradas y normaliza la distribución de integrinas CD26 e ICAM1 en la unión dermoepidérmica. ${ }^{4,13}$ 
Se ha propuesto la deficiencia de vitamina $\mathrm{D}$ como un desencadenante ambiental para trastornos de la inmunidad, entre los que destacan psoriasis y artritis psoriásica. ${ }^{14}$

El uso de análogos de vitamina D modula los marcadores de proliferación epidérmica, antígeno nuclear celular y Ki-67. Además, inhibe la proliferación de los queratinocitos disminuyendo niveles de defensina humana $\beta 2$ y 3, así como IL-17A, IL-17F, IL-8, IL-2, IL-6 e IFN- $\gamma$. Asimismo, tiene un efecto en la inducción de la diferenciación por los niveles de calcio intracelular., ${ }^{4,14}$ La combinación de calcipotriol con esteroides tópicos reduce el número los linfocitos CD4 y CD8, células de memoria TH12 y Th22.

Se ha demostrado una relación entre los niveles séricos de vitamina $\mathrm{D}$ y la gravedad de la psoriasis, sugiriendo que elevar los niveles séricos de vitamina $\mathrm{D}$ a través de la suplementación es benéfico. Ingram y su equipo evaluaron la suplementación oral a dosis de 100,000 UI por mes (equivalentes a $3340 \mathrm{UI} /$ día) con una dosis inicial de 200,000 UI independientemente del nivel sérico inicial, encontrando una relación inversa entre el PASI y los niveles séricos de vitamina $D$; sin embargo, no fue posible establecer un beneficio directo de esta acción. ${ }^{10}$ Debido a la relación estrecha entre los niveles de vitamina $\mathrm{D}$ y la psoriasis; diversos estudios recomiendan su monitorización al diagnóstico y durante la evolución de la enfermedad y suplementar al demostrar deficiencia de ésta. ${ }^{14}$

\section{Pustulosis palmoplantar}

La pustulosis palmoplantar es una enfermedad crónica, recurrente y refractaria caracterizada por pústulas estériles en palmas y plantas, que afecta al acrosiringio, donde se forman estas pústulas y comparte mecanismos fisiopatológicos con la psoriasis, como la elevación de IL17. El maxacalcitol, un análogo de vitamina D3, induce la inhibición de IL-8 e IL-6 y disminuye la proliferación de linfocitos. Aplicado vía tópica cada 12 horas por ocho semanas produce mejoría del eritema, pústulas y escamas, siendo efectivo como tratamiento de primera línea para esta dermatosis. ${ }^{15}$

\section{Dermatitis atópica}

La vitamina $\mathrm{D}$ se ha relacionado con disminución de la gravedad clínica de la dermatitis atópica por producir reducción de la respuesta inflamatoria, aumento en la actividad de péptidos antimicrobianos (AMP) y mantiene la actividad de barrera. A su vez, promueve la producción de catelidicina, filagrina y lípidos para la formación adecuada del estrato córneo, lo que previene la infección por virus herpes simple en el eccema herpético. ${ }^{16}$ La deficiencia de vitamina $D$ en pacientes con dermatitis atópica incrementa la susceptibilidad a infecciones por Staphylococcus aureus y Malassezia furfur por disminución de los niveles de AMP y alteración de la función de barrera. ${ }^{17}$ Del mismo modo, se han descubierto polimorfismos en el VDR en pacientes con dermatitis atópica en comparación con pacientes control. Diversos estudios indican que la suplementación con 1,600 UI al día apoya el tratamiento de la dermatitis atópica, ${ }^{6,18}$ ya que se considera segura y eficaz en pacientes con formas graves, particularmente en aquéllos con enfermedad persistente o refractaria a tratamiento. ${ }^{17}$

\section{Ictiosis}

La ictiosis es un trastorno de la queratinización condicionado por la diferenciación anormal del queratinocito y la descamación temprana de la epidermis. La vitamina $\mathrm{D}$ ha sido estudiada por su rol en la maduración y la inhibición de la proliferación de queratinocitos que provoca una mejoría en la función de barrera en la piel, mecanismo posible por la expresión de genes de respuesta de vitamina D como involucrina, peptidil arginina, transglutaminasa 1, calicreína e inhibidores de proteinasa B. En un estudio realizado por Gomathy Sethuraman y colaboradores se observó excelente respuesta clínica mejorando la descamación y la rigidez posterior a suplementación de un esquema intensivo con 60,000 UI al día por 10 días y posteriormente mantenimiento con $600 \mathrm{UI}$ de vitamina $\mathrm{D}$ vía oral todos los días. El resultado se observa desde el día cinco con mejoría progresiva hasta la completa integridad de la epidermis al mes de consumo en pacientes con deficiencia de vitamina D sérica. ${ }^{19}$

\section{Poroqueratosis}

La poroqueratosis es un grupo de trastornos de la queratinización de etiología incierta. Para su tratamiento se han utilizado los análogos de vitamina D tópicos con mejoría clínica de la poroqueratosis actínica superficial diseminada con o sin pigmentación residual leve. Aunque se desconoce el mecanismo de acción terapéutico de la vitamina D, se relaciona con la modulación de la diferenciación de los queratinocitos, proliferación e inflamación. ${ }^{20}$

\section{Cáncer de piel}

En estudios in vitro se ha confirmado que el VDR se encuentra en los queratinocitos malignos y no malignos, 
siendo protector de la acción mutágena de la radiación UV. Las teorías de la etiopatogenia de la vitamina D en el cáncer de piel, tanto melanoma como no melanoma, se ha asociado a cambios en la gravedad del cáncer relacionado con el nivel sérico de la misma. ${ }^{6}$

Un estudio reciente realizado por Liang y su equipo demostró asociación entre la deficiencia de vitamina D y el incremento de riesgo de cáncer de piel no melanoma. ${ }^{21}$ La radiación UV promueve carcinogénesis al inducir fotodaño en los queratinocitos debido a la formación de dímeros de ciclobutano pirimidina, inmunosupresión local y mecanismos de estrés oxidativo. Estudios in vitro han demostrado que la vitamina $\mathrm{D}$ reduce los dímeros de ciclobutano primidina, disminuye la apoptosis de los queratinocitos inducida por UVB y provoca la producción de metalotioneína, un antioxidante. El efecto de la vitamina D es dosis dependiente. ${ }^{22}$

\section{Queratosis actínicas}

La queratosis actínica es la etapa temprana de la transformación maligna de los queratinocitos, con posible progresión a carcinoma epidermoide. La combinación de calcipotriol con 5-fluorouracilo vía tópica previene el desarrollo de cáncer. La linfopoyetina del estroma tímico (LET) es un inductor potente de inmunidad antitumoral en la piel. Actualmente, se ha estudiado en modelos murinos que bloquean el desarrollo de cáncer al aumentar el reclutamiento de células T CD4 + en la piel. La expresión de la linfopoyetina y su efecto inmune son inducidos por el calcitriol, análogo de vitamina D.

Cunningham y colaboradores realizaron un estudio aleatorizado, doble ciego en el cual determinaron la eficacia y seguridad de la combinación de calcipotriol con 5-FU para el tratamiento de las queratosis actínicas. En 132 pacientes se utilizó calcipotriol al 0.005\% con 5-FU al $5 \%$ en 65 pacientes, mientras que 67 pacientes recibieron la fórmula de petrolato con 5-FU al 5\%. La aplicación se realizaba cada 12 horas por cuatro días. Se comprobó la eficacia superior en pacientes tratados con calcipotriol y 5- FU, de los cuales 82\% mejoraron, mientras que sólo $11 \%$ de los pacientes tratados con petrolato y 5 -FU refirieron mejoría. Los eventos adversos reportados fueron únicamente locales y de intensidad leve.

La respuesta inmunitaria que se observa es similar a un rechazo de aloinjerto de piel con presencia de HLA clase II. La linfopoyetina muestra una respuesta inmunitaria de memoria que prolonga el efecto antitumoral en la piel tratada. Debido a estos resultados, la aplicación de calcipotriol con 5-FU por cuatro días es más efectiva que los tratamientos tópicos aprobados en la actualidad para las queratosis actínicas. ${ }^{23}$

\section{Melanoma}

Estudios retrospectivos indican una asociación entre la deficiencia de vitamina $\mathrm{D}$ y un peor pronóstico en melanoma. La vitamina $D$ inhibe la síntesis del ADN en queratinocitos epidérmicos y en células de melanoma, siendo estos últimos más sensibles a su efecto antiproliferativo y de pro-diferenciación por activación del VDR. Esta señalización suprime la actividad de IL-8 inducida por el TNF $\alpha$, disminuyendo su efecto proinflamatorio. ${ }^{24}$ De la misma manera, inhibe el crecimiento y promueve la apoptosis de células tumorales o sus progenitores. Por último, funciona como mecanismo protector que aumenta la reparación de $\mathrm{ADN}$, protege ante las especies reactivas de oxígeno y activa los inmunomoduladores. A mayor nivel sérico de vitamina $D$ existe mayor probabilidad de que el melanoma se encuentre sin ulceración y con una tasa mitótica menor, indicando un rol protector de la vitamina D en el melanoma. ${ }^{25}$

El índice de Breslow en melanoma es el indicador pronóstico más importante. Se ha determinado que una disminución en el nivel de vitamina $\mathrm{D}$ se relaciona con un incremento en la profundidad de Breslow y con un estadio avanzado al momento del diagnóstico muestra un peor pronóstico. Wyatt y colaboradores realizaron un estudio en 100 pacientes con diagnóstico reciente de melanoma en el que se examinó el antecedente de exposición solar y el nivel sérico de vitamina $\mathrm{D}$ basal en relación con indicadores de pronóstico histopatológico como el índice de Breslow, ulceración, el nivel de Clark y la presencia de actividad mitótica. Concluyen que el tener deficiencia de vitamina D sérica al momento del diagnóstico de melanoma está asociado a un riesgo más alto de presentar mayor Breslow y, por lo tanto, un peor pronóstico. ${ }^{26} \mathrm{La}$ deficiencia de la vitamina $\mathrm{D}$ aumenta la tumorogénesis, la invasión y la metástasis temprana. La expresión del VDR se encuentra inversamente relacionada con la progresión tumoral, siendo relevante en la prevención de la progresión de melanoma mejorando la supervivencia. Por lo tanto, la evidencia apoya la hipótesis de que variantes en la señalización de vitamina D desempeñan un rol importante en la supervivencia del melanoma. ${ }^{24,27}$ Se concluye que la terapia con vitamina D vía oral a dosis de 50,000 UI por mes puede ser utilizada como factor adyuvante en el tratamiento del melanoma, ya que los cambios en la concentración de vitamina $\mathrm{D}$ posterior al diagnóstico y durante el seguimiento son más importantes y pueden ser 
un marcador de pronóstico, incluso aumentando la eficacia terapéutica de los medicamentos de primera línea. ${ }^{28}$

\section{Urticaria}

La prevalencia de deficiencia de vitamina $\mathrm{D}$ es mayor en pacientes con urticaria crónica en comparación con la urticaria aguda. El nivel de deficiencia se relaciona directamente con la gravedad clínica de la urticaria crónica. Woo y colaboradores realizaron determinaciones séricas de vitamina $\mathrm{D}$ en 72 pacientes con diagnóstico de urticaria crónica y 26 pacientes con urticaria aguda. Se observó que el nivel más bajo se presentó en los pacientes con urticaria crónica con un promedio de $11.86 \mathrm{ng} / \mathrm{mL}$, comparado con el grupo de urticaria aguda con $14.12 \mathrm{ng} / \mathrm{mL}$. El nivel sérico de vitamina $\mathrm{D}$ muestra una relación inversa con la gravedad de la enfermedad y la duración ésta. ${ }^{29}$ Los niveles séricos de vitamina $D$ menores de $10 \mathrm{ng} / \mathrm{mL}$ se asocian a urticaria autoinmune. En un estudio realizado por Goets se demostró que la suplementación vía oral de vitamina D a una dosis de 50,000 UI por semana disminuye los síntomas de urticaria crónica y aumenta la calidad de vida desde el primer mes de seguimiento. ${ }^{30}$ Por lo tanto, la suplementación puede ser un complemento a la primera línea de tratamiento de urticaria crónica. ${ }^{31}$

\section{Verrugas vulgares}

En un estudio realizado por Aktas y su equipo se aplicó la vitamina $D$ intralesional en pacientes con verrugas plantares. Se preparó una solución inyectable de $7.5 \mathrm{mg} / \mathrm{dL}$ de vitamina $\mathrm{D}$ y se administró $0.2 \mathrm{~mL}$ de dicha solución en la base de la verruga, posterior a la aplicación de 0.1 $\mathrm{mL}$ de pilocarpina. Se obtuvo una tasa de remisión de $70 \%$ total, posterior a un mes de tratamiento con una sola aplicación. 15\% de los pacientes remitió posteriormente con una segunda dosis, teniendo una tasa de mejoría de 85\%. En el seguimiento seis meses posterior a remisión con una o dos dosis de vitamina D se observó que $80 \%$ se mantuvo asintomático. No se reportaron eventos adversos. La indicación de aplicación de este medicamento es la falta de respuesta a tratamiento de primera línea. ${ }^{32}$

\section{Lupus eritematoso sistémico (LES)}

Estudios in vitro con vitamina $\mathrm{D}$ sustentan la función inhibitoria de la producción de IgG anti-ADN de doble cadena, así como la disminución de la maduración de células dentríticas, la regulación de la respuesta de linfocitos B y T, y el aumento de la función de los fagocitos para eliminar los complejos inmunes y cuerpos apoptóticos, que interfieren con la aparición y persistencia de la enfermedad.

Los polimorfismos del VDR se han implicado con la susceptibilidad a las manifestaciones cutáneas con un aumento en el daño al ADN inducido por la radiación UV, en específico la aparición de eritema malar por fotosensibilidad, Se ha descrito una mutación en el gen rs11168268 relacionada con una afección cutánea más agresiva. ${ }^{33,34}$ Teniendo en cuenta su función inmune, el monitoreo y tratamiento con la vitamina $\mathrm{D}$ en el paciente con LES es una opción para el control de síntomas. La deficiencia de la vitamina $\mathrm{D}$ en LES se relaciona con mayor edad y duración de la enfermedad, siendo terapéutica útil por su efecto en la reducción de IFN $\gamma$ e IL-17. Es recomendable la suplementación de 1,400 UI de vitamina D al día por 40 días y posterior mantenimiento con 800 UI al día por un año en pacientes con nivel sérico menor de $30 \mathrm{ng} / \mathrm{mL} .{ }^{35-37}$

\section{Vitíligo}

Se sabe que existe una expresión elevada de IL-17 (citocina proinflamatoria) en tejido y sangre periférica de pacientes con vitíligo, así como niveles disminuidos de vitamina D sérica comparados contra sanos. Al mismo tiempo, se ha propuesto que la vitamina $\mathrm{D}$ posee un efecto inmunomodulador en células TH-17. ${ }^{38}$ Los niveles bajos de vitamina $\mathrm{D}$ alteran las vías $\mathrm{TH}-1$ y $\mathrm{TH}-17$, contribuyendo en la patogénesis del vitiligo. ${ }^{39}$

Estudios experimentales han demostrado que al cultivar melanocitos en medios enriquecidos con vitamina $D$, incrementan su tamaño, nivel de tirosinasa y la longitud y número de dendritas. ${ }^{40}$

Karagüzel y colaboradores demostraron que al suplementar vitamina D vía oral de 1,500-3,000 UI al día en pacientes con vitíligo, que recibían terapia tópica con inhibidores de calcineurina, mostraban una tasa más rápida de repigmentación, comparados con aquéllos que sólo recibían tratamiento tópico. ${ }^{41}$

\section{Micosis fungoides}

Las células aberrantes prolongan la estimulación antigéni$\mathrm{ca}$, lo que produce linfocitos $\mathrm{T}$ deficientes y resistentes a la apoptosis mediada por Fas y que liberan citocinas Th2. Estas células alteradas poseen polimorfismos del VDR. El mecanismo por el cual la deficiencia de vitamina D se encuentra dentro de la patogénesis se explica porque ésta promueve la disminución de citocinas TH1, y regula la producción de catelicidina y AMP. Por lo tanto, la deficiencia promueve la colonización por Staphylococcus 
aureus, desencadena la estimulación antigénica y la activación de células $T$, aumentando la producción de células aberrantes. Un nivel sérico disminuido se ha relacionado con peor pronóstico. Se recomienda la suplementación de 50,000 UI dos veces por semana en conjunto con la terapéutica habitual. ${ }^{42}$

\section{Displasia ectodérmica}

La displasia ectodérmica es un trastorno genético heterogéneo caracterizado por desarrollo anormal de estructuras del ectodermo como pelo, dientes, uñas y glándulas sudoríparas. La estructura más frecuentemente afectada es el pelo. Xie y colaboradores reportaron que el VDR es necesario para la señalización del queratinocito por la vía WNT, donde la ausencia de dicha vitamina produce disfunción del ciclo del pelo. ${ }^{43}$ Rossi y su equipo recomiendan comenzar tratamiento con cetirizina tópica y vitamina D vía oral a dosis de 1,000 UI diarias por un año para la mejoría en el grosor, color y densidad de pelo. ${ }^{44}$

\section{Acné}

Estudios in vitro apoyan la teoría que establece que la vitamina $\mathrm{D}$ forma parte de la etiopatogenia de la enfermedad debido a la presentación del VDR en sebocitos modulando la producción de citocinas. La deficiencia de la vitamina D se correlaciona con mayor gravedad, al igual que en otras dermatosis inflamatorias.

El mecanismo de acción es disminuir la expresión de marcadores inflamatorios como IL-6, IL-8 y metaloproteinasa 9 y promover la proliferación y diferenciación de queratinocitos y sebocitos con propiedades anticomedogénicas. Al reducir la expresión de IL-17, inhibe la diferenciación inducida por C. acnes. Su actividad antimicrobiana se relaciona con la producción de IL-37. ${ }^{45}$ El tratamiento con isotretinoína por tres meses aumenta el nivel de vitamina D sérica, ${ }^{46}$ así como la suplementación con 1,000 UI al día vía oral produce mejoría clínica del acné ${ }^{46}$

\section{Alopecia androgenética}

Un estudio realizado por Rasheed y colaboradores afirma que la deficiencia de vitamina $\mathrm{D}$ se asocia a pérdida de pelo en pacientes con alopecia androgenética de patrón femenino, por lo que sugiere la determinación al momento del diagnóstico y la suplementación en caso de deficiencia. ${ }^{47}$ El tratamiento con análogos de vitamina $D$ se asoció a la formación folicular y aumento en la expresión de queratinas Ha7, Ha8 y Hb3. De la misma manera, estudios in vitro indican que el VDR es fundamental en la preservación del cabello posterior al nacimiento. En la fase de catágeno, la concentración del VDR se encuentra elevado, aumentando la diferenciación de los queratinocitos y estimulando el ciclo del pelo. ${ }^{48}$

\section{Alopecia areata}

Se ha demostrado expresión del VDR en la vaina radicular externa, el bulbo del folículo piloso y glándula sebácea. EI VDR es necesario para el equilibro epidérmico, ya que inhibe la expresión de marcadores inflamatorios como TNF $\alpha$, IL-6 e IL-8. La ausencia del receptor se relaciona con una disminución del crecimiento del pelo y reducción de la vía Wnt/beta, produciendo alopecia areata. El estudio realizado por Unal y colegas refiere la relación inversa entre el nivel de vitamina D y la duración de la alopecia areata con ausencia de inflamación. ${ }^{47}$ El estudio considera que la suplementación en pacientes con alopecia areata permite el crecimiento del pelo, disminuye la recurrencia y mantiene la remisión de ésta. Se propone también la medición como indicador de la actividad o recaída de la enfermedad. En el caso específico de niños, se recomienda la suplementación como tratamiento complementario. ${ }^{49-51}$

\section{Pie diabético}

La vitamina $\mathrm{D}$ permite el crecimiento y la diferenciación de queratinocitos y fibroblastos al modular los factores de crecimiento. Un nivel sérico adecuado de vitamina $\mathrm{D}$ es esencial para la cicatrización normal de cualquier herida. La deficiencia de vitamina D se correlaciona con complicaciones microvasculares y macrovasculares propias de la diabetes mellitus. La vitamina D produce AMP en queratinocitos de las úlceras de pie diabético. Estudios en ratones confirman que la aplicación de vitamina $D$ promueve una cicatrización más rápida y adecuada. Razzaghi y colaboradores estudiaron la suplementación con 50,000 UI de vitamina D vía oral cada dos semanas por 12 semanas, con mejoría en la cicatrización de heridas por pie diabético y en el control de glucosa sérica..$^{52,53}$

\section{Lepra}

Los macrófagos son parte del sistema inmunitario innato, que se relaciona con la respuesta antimicrobiana dependiente de vitamina D para la destrucción de patógenos. Elliot y su equipo afirman que un nivel suficiente de vitamina $\mathrm{D}$ previo a la infección, reduce efectivamente la viabilidad del patógeno en el macrófago. Por lo tanto, 
la capacidad del macrófago para producir una respuesta efectiva en contra de la micobacteria intracelular depende de su habilidad de inducir la producción de catelicidinas por la vía de señalización dependiente de vitamina D.

La segunda vía por la cual la vitamina D promueve la función del macrófago es a través del aumento en la diferenciación del monocito al macrófago activado, que tiene capacidad de producción de IL-15, aumentando así su función antimicrobiana. Por último, una tercera vía por la cual la vitamina $\mathrm{D}$ es elemental para la respuesta inmunitaria es la inducción de la diferenciación del monocito a célula dendrítica a través del factor estimulante de colonias granulocito-macrófago. Por lo tanto, es necesario un nivel adecuado de vitamina D mayor de $30 \mathrm{ng} / \mathrm{mL}$ para mantener y promover una respuesta antimicrobiana adecuada frente al Mycobacterium leprae. ${ }^{54}$

\section{CONCLUSIÓN}

La vitamina $\mathrm{D}$ es una hormona relacionada con la producción y modulación de interleucinas y citocinas res- ponsables de la respuesta inmunitaria frente a patógenos exógenos. Las alteraciones en las mismas son causa y consecuencia de enfermedades autoinmunes, y tienen como común denominador deficiencia de vitamina D. Debido a su rol en la inflamación, la vitamina D se asocia a diversas enfermedades dermatológicas. Se recomienda realizar un tamizaje al diagnóstico de las enfermedades vinculadas con la deficiencia de vitamina D y suplementar para mejorar el pronóstico y la calidad de vida de los pacientes. Consideramos que la suplementación de vitamina $\mathrm{D}$ es útil para mantener la homeostasis celular de la piel.

\section{BIBLIOGRAFÍA}

1. Reichrath J, Saternus R, Vogt T. Endocrine actions of vitamin D in skin: Relevance for photocarcinogenesis of non-melanoma skin cancer, and beyond. Mol Cell Endocrinol. 2017; 453: 96102.

2. Holick MF. Vitamin D and bone health. J Nutr. 1996; 126: 1159S-1164S.

3. Kechichian E, Ezzedine K. Vitamin D and the skin: an update for dermatologists. Am J Clin Dermatol. 2018; 19: 223-235.

4. Mostafa WZ, Hegazy RA. Vitamin D and the skin: focus on a complex relationship: a review. J Adv Res. 2015; 6: 793-804.

5. Lim A, Shayan R, Varigos G. High serum vitamin D level correlates with better prognostic indicators in primary melanoma: A pilot study. Australas J Dermatol. 2018; 59:182-187.

6. Wadhwa B, Relhan V, Goel K, Kochhar AM, Garg VK. Vitamin D and skin diseases: A review. Indian J Dermatol Venereol Leprol. 2015; 81: 344-355.

7. Adams JS, Ren J, Liu PT, Chun RF, Lagishetty V, Gombart AF et al. Vitamin d-directed rheostatic regulation of monocyte antibacterial responses. J Immunol. 2009; 182: 4289-4295.

8. Maleki M, Nahidi Y, Azizahari S, Meibodi NT, Hadianfar A. Serum $25-\mathrm{OH}$ vitamin $\mathrm{D}$ level in psoriatic patients and comparison with control subjects. J Cutan Med Surg. 2016; 20: 207-210.

9. Scott JF, Das LM, Ahsanuddin S, Qiu Y, Binko AM, Traylor ZP et al. Oral vitamin D rapidly attenuates inflammation from sunburn: an interventional study. J Invest Dermatol. 2017; 137 (10): 2078-2086.

10. Scott JF, Lu KQ. Vitamin D as therapeutic option for sunburn: clinical and biologic implications. DNA Cell Biol. 2017; 36: 879882.
11. Ingram MA, Jones MB, Stonehouse $W$, Jarret $P$, Scragg $R$, Mugdrige $\mathrm{O}$ et al. Oral vitamin D3 supplementation for chronic plaque psoriasis: a randomized, double-blind, placebocontrolled trial. J Dermatol Treat. 2018; 22: 1-10.

12. Filoni A, Vestita M, Congedo M, Giudice G, Tafuri S, Bonamonte D. Association between psoriasis and vitamin D: Duration of disease correlates with decreased vitamin $D$ serum levels: An observational case-control study. Medicine (Baltimore). 2018; 97 (25): e11185.

13. Kubin ME, Kokkonen N, Palatsi R, Hagg PM, Vayrynen KP, Haapasaari KM et al. Clinical efficiency of topical calcipotriol/ betamethasone treatment in psoriasis relies on suppression of the inflammatory TNF $\alpha$-IL-23-IL-17 Axis. Acta Derm Venereol. 2017; 97: 449-455.

14. Al-Dhubaibi MS. Association between vitamin D deficiency and psoriasis: an exploratory study. Int J Health Sci (Qassim). 2018; 12 (1): 33-39.

15. Umezawa Y, Nakagawa H, Tamaki K. Phase III clinical study of maxacalcitol ointment in patients with palmoplantar pustulosis: a randomized, double-blind, placebo-controlled trial. J Dermatol. 2016; 43 (3): 288-293.

16. Albenali LH, Danby S, Moustafa M, Brown K, Chittocl J, Shackley $F$ et al. Vitamin $D$ and antimicrobial peptide levels in patients with atopic dermatitis and atopic dermatitis complicated by eczema herpeticum: A pilot study. J Allergy Clin Immunol. 2016; 138: 1725-1719.

17. Dogru $M$. Is vitamin $D$ level associated with the natural course of atopic dermatitis? Allergol Immunopathol (Madr). 2018; 46 (6): 546-551. 
18. Vieira BL, Lim NR, Lohman ME, Lio PA. Complementary and alternative medicine for atopic dermatitis: an evidence-based review. Am J Clin Dermatol. 2016; 17: 557-581.

19. Sethuraman G, Marwaha RK, Challa A, Yenamandra VK, Ramakrishnan L, Thulkar S et al. Vitamin D: a new promising therapy for congenital ichthyosis. Pediatrics. 2016; 137 (1).

20. Weidner T, Illing T, Miguel D, Elsner P. Treatment of porokeratosis: a systematic review. Am J Clinic Dermatol. 2017; 18: 435-449.

21. Liang G, Nan H, Qureshi AA, Han J. Pre-diagnostic plasma 25-hydroxyvitamin $D$ levels and risk of non-melanoma skin cancer in women. PLoS One. 2012; 7 (4): e35211.

22. Cerman AA, Karabay EA, Altunay IK, Cesur SK. Vitamin D levels in actinic keratosis: a preliminary study. An Bras Dermatol. 2018; 93: 535-538.

23. Cunningham TJ, Tabacchi M, Eliane JP, Tuchayi SM, Manivasagam $\mathrm{S}$, Mirzaalian $\mathrm{H}$ et al. Randomized trial of calcipotriol combined with 5-fluorouracil for skin cancer precursor immunotherapy. J Clin Invest. 2016; 127 (1): 106-116.

24. Orlow I, Reiner AS, Thomas NE, Roy P, Kanetsky PA, Luo $L$ et al. Vitamin $D$ receptor polymorphism and survival in patients with cutaneous melanoma: a population- based study. Carcinogenesis. 2016; 37: 30-38.

25. Paolino G, Panetta C, Cota C, Didona D, Moliterni E, Di Mattia $C$ et al. Vitamin $D$ receptor immunohistochemistry variability in sun-exposed and non-sun-exposed melanomas. Melanoma Res. 2017; 27: 17-23.

26. Wyatt C, Lucas RM, Hurst C, Kimlin MG. Vitamin D deficiency at melanoma diagnosis is associated with higher Breslow thickness. PLoS One. 2015; 10 (5): e0126394.

27. Cattaruzza MS, Pisani D, Fidanza L, Gandini S, Marmo G, Narcisi A et al. 25-Hydroxivitamin D serum levels and melanoma risk: a case control study and evidence synthesis of clinical epidemiological studies. Eur J Cancer Prev. 2019; 28 (3): 203-211.

28. Slominski AT; Brozyna AA, Skobowiat C, Zmijewski MA, Kim TK, Janjetovic $Z$ et al. On the role of classical and novel forms of vitamin $D$ in melanoma progression and management. J Steroid Biochem Mol Biol. 2018; 177: 159-170.

29. Woo YR, Jung KE, Koo DW, Lee JS. Vitamin D as a marker for disease severity in chronic urticaria and its possible role in pathogenesis. Ann Dermatol. 2015; 27 (4): 423-430.

30. Goetz DW. Idiopathic itch, rash, and urticaria/angioedema merit serum vitamin D evaluation: a descriptive case series. $W V$ Med J. 2011; 107 (1): 14-20.

31. Rather S, Keen A, Sajad P. Serum levels of 25-hydroxyvitamin $D$ in chronic urticaria and its association with disease activity: a case control study. Indian Dermatol Online J. 2018; 9: 170-174.

32. Aktas H, Ergin C, Demir B, Ekiz O. Intralesional vitamin D injection may be an effective treatment option for warts. J Cutan Med Surg. 2016; 20: 118-122.

33. Hersh AO, Arkin LM, Prahalad S. Immunogenetics of cutaneous lupus erythematosus. Curr Opin Pediatr. 2016; 28: 470-475.

34. García M, Mendoza C, Etchegaray I, Soto P, Jiménez E, Robles $V$ et al. Insuficiencia y deficiencia de vitamina $D$ en pacientes mexicanas con lupus eritematoso sistémico: prevalencia y relación con actividad de la enfermedad. Reumatol Clin. 2017; 13: $97-101$

35. Cutillas-Marco E, Marquina-Vila A, Grant WB et al. Vitamin D and cutaneous lupus erythematosus: effect of vitamin $D$ replacement on disease severity. Lupus. 2014; 23 (7): 615-623.

36. Chang J, Weth VP. Therapeutic options for cutaneous lupus erythematosus: recent advances and future prospects. Expert Rev Clin Immunol. 2017; 12: 1009-1021.
37. Mok CC, Bro ET, Ho LY, Singh RJ, Jannetto PJ. Serum 25-hydroxyvitamin D3 levels and flares of systemic lupus erythematosus: a longitudinal cohort analysis. Clin Rheumatol. 2018; 37 (10): 2685-2692.

38. Aly D, Mohammed F, Sayed K, Gawdat H, Mashaly H, Abdel Hay $R$ et al. Is there a relation between vitamin $D$ and interleukin-17 in vitiligo? A cross-sectional Study. Dermatology. 2017; 233: 413-418.

39. Doss RW, El-Rifaie AA, Gohary YM, Rashed LA. Vitamin D receptor expression in vitiligo. Indian J Dermatol. 2015; 60: 544-548.

40. Tomita $\mathrm{Y}$, Torinuki W, Tagami H. Stimulation of human melanocytes by vitamin D3 possibly mediates skin pigmentation after sun exposure. J Invest Dermatol. 1988; 90: 882e4.

41. Karagüzel G, Sakarya NP, Bahadir S, Yaman S, Okten A. Vitamin $D$ status and the effects of oral vitamin $D$ treatment in children with vitiligo: A prospective study. Clin Nutr. 2016; 15: 28-31.

42. Rasheed H, Hegazy RA, Gawdat HI, Mehaney DA, Kamel MM, Fawzy MM et al. Serum vitamin D and vitamin d receptor gene polymorphism in mycosis fungoides patients: a case control study. PLoS One. 2016; 11 (6): e0158014.

43. Xie Z, Komuves L, Yu QC et al. Lack of the vitamin D receptor is associated with reduced epidermal differentiation and hair follicle growth. J Invest Dermatol. 2002; 118: 11-16.

44. Rossi A, Miraglia E, Fortuna MC, Calvieri S, Giustini S. Topical cetirizine and oral vitamin D: A valid treatment for hypotrichosis caused by ectodermal dysplasia. J Eur Acad Dermatol Venereol. 2017; 31: 367-370.

45. Lim SK, Ha JM, Lee YH, Lee Y, Seo YJ, Kim CD et al. Comparison of vitamin $\mathrm{D}$ levels in patients with and without acne: a case-control study combined with a randomized controlled trial. PLoS One. 2016; 11 (8): e0161162.

46. El-Hamd MA, EI Taieb MA, Ibrahim HM, Aly SS. Vitamin D levels in acne vulgaris patients treated with oral isotretinoin. $J$ Cosmet Dermatol. 2019; 18 (1): 16-20.

47. Rasheed H, Mahgoub D, Hegazy R, El-Komy M, Abdel Hay R, Hamid MA et al. Serum ferritin and vitamin $D$ in female hair loss: do they play a role? Skin Pharmacol Physiol. 2013; 26: 101-107.

48. Banihashemi M, Nahidi Y, Meibodi NT, Jarah L, Dolatkhah M. Serum vitamin D3 level in patients with female pattern hair loss. Int J Trichology. 2016; 8: 116-120.

49. Unal M, Gonulalan $\mathrm{G}$. Serum vitamin $\mathrm{D}$ level is related to disease severity in pediatric alopecia areata. J Cosmet Dermatol. 2018; 17: 101-104.

50. Gade VKV, Mony A, Munisamy M, Chandrashekar L, Rajappa M. An investigation of vitamin D status in alopecia areata. Clin Exp Med. 2018; 18 (4): 577-584.

51. Lee S, Kim BJ, Lee CH, Lee WS. Increased prevalence of vitamin $\mathrm{D}$ deficiency in patients with alopecia areata: a systematic review and meta-analysis. J Eur Acad Dermatol Venereol. 2018; 32: 1214-1221.

52. Razzaghi R, Pourbagheri H, Momen-Heravi M, Bahmani F, Shadi $\mathrm{J}$, Soleimani $Z$ et al. The effects of vitamin D supplementation on wound healing and metabolic status in patients with diabetic foot ulcer: A randomized, double-blind, placebo-controlled trial. J Diabetes Complications. 2017; 31 (4): 766-772.

53. Fledkamo J, Jungheim K, Schott M, Jacobs B, Roden M. Severe vitamin D3 deficiency in the majority of patients with diabetic foot ulcers. Hom Metab Res. 2018; 50: 615-619.

54. Kim EW, Teles RMB, Haile S, Liu PT, Modlin RL. Vitamin D status contributes to the antimicrobial activity of macrophages against Mycobacterium leprae. PLoS Negl Trop Dis. 2018; 12 (7): e0006608. 